\title{
ON THE BORDISM RING OF COMPLEX PROJECTIVE SPACE
}

\author{
CLAUDE SCHOCHET
}

\begin{abstract}
The bordism ring $M U_{*}\left(C P^{\infty}\right)$ is central to the theory of formal groups as applied by D. Quillen, J. F. Adams, and others recently to complex cobordism. In the present paper, rings $E_{*}(C P \infty)$ are considered, where $E$ is an oriented ring spectrum, $R=\pi_{*}(E)$, and $p R=0$ for a prime $p$. It is known that $E_{*}(C P \infty)$ is freely generated as an $R$-module by elements $\left\{\beta_{r} \mid r \geqq 0\right\}$. The ring structure, however, is not known. It is shown that the elements $\left\{\beta_{p^{r}} \mid r \geqq 0\right\}$ form a simple system of generators for $E_{*}\left(C P^{\infty}\right)$ and that $\beta_{p^{r}}^{p^{r}} \equiv s^{p^{r}} \beta_{p^{r}} \bmod \left(\beta_{1}, \cdots, \beta_{p^{r-1}}\right)$ for an element $s \in R$ (which corresponds to $\left[C P^{p-1}\right]$ when $E=M U Z_{p}$ ). This may lead to information concerning $E_{*}(K(Z, n))$.
\end{abstract}

1. Introduction. Let $E$ be an associative, commutative ring spectrum with unit, with $R=\pi_{*} E$. Then $E$ determines a generalized homology theory $E_{*}$ and a generalized cohomology theory $E^{*}$ (as in G. W. Whitehead [5]). Following J. F. Adams [1] (and using his notation throughout), assume that $E$ is oriented in the following sense:

There is given an element $x \in \tilde{E}^{*}\left(C P^{\infty}\right)$ such that $\tilde{E}^{*}\left(S^{2}\right)$ is a free $R$ module on $i^{*}(x)$, where $i: S^{2}=C P^{1} \rightarrow C P^{\infty}$ is the inclusion.

(The Thom-Milnor spectrum $\boldsymbol{M U}$ which yields complex bordism theory and cobordism theory satisfies these hypotheses and is of seminal interest.) By a spectral sequence argument and general nonsense, Adams shows:

(1.1) $E^{*}\left(C P^{\infty}\right)$ is the graded ring of formal power series $R[[x]]$.

(1.2) The map $m: E^{*}\left(C P^{\infty}\right) \rightarrow E^{*}\left(C P^{\infty} \times C P^{\infty}\right)$ induced by the group multiplication on $C P^{\infty}=K(Z, 2)$ gives $E^{*}\left(C P^{\infty}\right)$ and $E_{*}\left(C P^{\infty}\right)$ the structure of commutative, cocommutative Hopf algebras over $R$.

Writing the coproduct $m: R[[x]] \rightarrow R\left[\left[x_{1}, x_{2}\right]\right]$ by $m(x)=\mu\left(x_{1}, x_{2}\right)=$ $\sum a_{i j} x_{1}^{i} x_{2}^{j},(1.2)$ implies

(1.3) $m$ is $R$-linear and satisfies the equations

$$
\begin{gathered}
\mu\left(x_{1}, 0\right)=x_{1}, \quad \mu\left(0, x_{2}\right)=x_{2}, \\
\mu\left(x_{1}, \mu\left(x_{2}, x_{3}\right)\right)=\mu\left(\mu\left(x_{1}, x_{2}\right), x_{3}\right), \\
\mu\left(x_{1}, x_{2}\right)=\mu\left(x_{2}, x_{1}\right) .
\end{gathered}
$$

Received by the editors April 14, 1972.

AMS (MOS) subject classifications (1970). Primary 57D90, 57F05; Secondary 14L05.

Key words and phrases. Complex bordism, complex cobordism, oriented spectrum, graded formal group, Hopf algebra over a ring. 
Condition (1.3) is precisely the statement that $m$ is a formal product and that $\left(E^{*}\left(C P^{\infty}\right), m\right)$ is a formal group. Recent work by Quillen [3] and others has indicated the great strength of formal group techniques in studying complex bordism and cobordism. It therefore seems reasonable to attain to a very firm grasp on the Hopf algebra $E_{*}\left(C P^{\infty}\right)$, which is central to bordism applications.

Let $\langle\rangle:, E^{*}\left(C P^{\infty}\right) \otimes E_{*}\left(C P^{\infty}\right) \rightarrow R$ be the Kronecker pairing. There are unique elements $\beta_{n} \in E_{*}\left(C P^{\infty}\right)$ such that $\left\langle x^{i}, \beta_{n}\right\rangle=\delta_{n}^{i}$. Adams proves:

(1.4) $E_{*}\left(C P^{\infty}\right)$ is a free $R$-module on generators $1=\beta_{0}, \beta_{1}, \cdots, \beta_{n}, \cdots$.

(1.5) The coproduct $\Psi$ on $E_{*}\left(C P^{\infty}\right)$ is determined by $\Psi\left(\beta_{n}\right)=$ $\sum_{i=0}^{n} \beta_{i} \otimes \beta_{n-i}$.

The remaining open problem is the expression of the algebra structure of $E_{*}\left(C P^{\infty}\right)$ in some reasonable way. This would be of some conceptual interest, and it would also be of practical interest, for example, in the computation of $E_{*}(K(Z, 3))$ via the Eilenberg-Moore spectral sequence with $E_{2}=\operatorname{Tor}_{E_{*}\left(C P^{\infty}\right)}(R, R)$.

Our approach to the problem is via restriction to

2. Hopf algebras of prime characteristic. Henceforth assume that $p R=0$ for a fixed prime $p$. (For example, one could take $\boldsymbol{E}$ to be the spectrum corresponding to the theory $M U_{*}\left(; Z_{p}\right)$, where $Z_{p}$ is the field of $p$ elements.)

(2.1) Definition. An augmented $R$-algebra $A$ is said to have the ordered set $y_{1}, y_{2}, \cdots, y_{n}, \cdots$ as a simple system of generators if the monomials

$$
\left\{y_{j_{1}}^{t_{1}} \cdots y_{j_{k}}^{t_{k}} \mid j_{1}<j_{2}<\cdots<j_{k} \text { and } 0<t_{i}<p\right\}
$$

form a free $R$-basis for $A$, and if for each $n$, only finitely many $y_{j}$ have degree $n$.

Let $Q A$ denote the $R$-module of indecomposable elements of $A$; i.e. $Q A=I A /(I A)^{2}$ where $I A$ is the kernel of the augmentation $A \rightarrow R$.

(2.2) MAIN Theorem. (a) The elements $\beta_{1}, \beta_{p}, \cdots, \beta_{p^{n}}, \cdots$ form $a$ free $R$-basis for $Q E_{*}\left(C P^{\infty}\right)$.

(b) $\beta_{p^{n}}^{p} \equiv s^{p^{n}} \beta_{p^{n}} \bmod \left(\beta_{1}, \cdots, \beta_{p^{n-1}}\right)$ where $s=\sum_{i=1}^{p-1} a_{i, 1}\left\langle x^{i}, \beta_{1}^{p-1}\right\rangle \in R$.

(c) If $\operatorname{deg}(x)<0$, then the elements $\beta_{1}, \beta_{p}, \cdots, \beta_{p^{n}}, \cdots$ form a simple system of generators for $E_{*}\left(C P^{\infty}\right)$.

The proof of the Main Theorem is simple and purely algebraic, resting upon a decomposition theorem for Hopf algebras of the type $E_{*}\left(C P^{\infty}\right)$ which is stated below and proved in [4]. We now present the algebraic setting for 
3. The Decomposition Theorem. Let $R$ be a graded commutative ring with $R_{n}=0$ if $n$ is negative, $p R=0$ for a prime integer $p$, and $x$ an indeterminate of nonpositive degree. Define $F$ to be the free $R$-module on generators $1=\beta_{0}, \beta_{1}, \cdots, \beta_{n}, \cdots$ with $\operatorname{deg}\left(\beta_{k}\right)=-\operatorname{deg}\left(x^{k}\right)$. Give $F$ the structure of an $R$-coalgebra via the Whitney coproduct

$$
\Psi\left(\beta_{n}\right)=\sum_{i=0}^{n} \beta_{i} \otimes \beta_{n-i}
$$

and define the Kronecker pairing $\langle\rangle:, R[[x]] \otimes F \rightarrow R$ by $\left\langle x^{i}, \beta_{n}\right\rangle=\delta_{n}^{i}$. Let $m: R[[x]] \rightarrow R\left[\left[x_{1}, x_{2}\right]\right]$ be a cocommutative formal product (satisfying (1.3)), so that $F$ becomes a commutative, cocommutative Hopf algebra over $R$.

Let $F_{0}$ be the $R$-subalgebra of $F$ generated by $\beta_{1}$. Direct calculation shows

$$
\beta_{1}^{p}=s \beta_{1} \text { in } F
$$

where $s=\sum_{i=1}^{p-1} a_{i, 1}\left\langle x^{i}, \beta_{1}^{p-1}\right\rangle \in R$. Hence $F_{0}$ is isomorphic as an $R$-algebra to the polynomial algebra generated over $R$ by $\bar{\beta}_{1}$ modulo the relation $\bar{\beta}_{1}^{p}=s \bar{\beta}_{1}$. In fact, the isomorphism is as Hopf algebras, if we assume $\bar{\beta}_{1}$ to be primitive.

Define $F_{n}$ to be the polynomial algebra generated over $R$ by $\bar{\beta}_{p^{n}}$ (where $\left.\operatorname{deg}\left(\bar{\beta}_{k}\right)=\operatorname{deg}\left(\beta_{k}\right)\right)$ modulo the relation

$$
\bar{\beta}_{p^{n}}=s^{p^{n}} \bar{\beta}_{p^{n}}
$$

with the Hopf algebra (over $R$ ) structure obtained by declaring $\bar{\beta}_{p^{n}}$ to be primitive.

(3.4) DeCOMPosition THEOREM. There exists a diagram of Hopf algebras and morphisms of Hopf algebras over $R$ :

$$
\begin{aligned}
& F_{0} \rightarrow F=G_{0} \\
& F_{1} \rightarrow \stackrel{\downarrow}{G_{1}} \\
& F_{2} \rightarrow \stackrel{\downarrow}{ } \rightarrow G_{2}
\end{aligned}
$$

such that for each $n \geqq 1$,

$$
\begin{aligned}
& \mathscr{A}_{n}: G_{n}=G_{n-1} / / F_{n-1}=G_{n-1} \otimes_{F_{n-1}} R \text { is the free } R \text {-module on generators } \\
& \quad 1=\beta_{0}, \beta_{p^{n}}, \beta_{2 p^{n}}, \cdots, \beta_{k p^{n}}, \cdots \text { (which are the images of } \beta_{k p^{n}} \in G_{0} \\
& \text { under projection), }
\end{aligned}
$$


$\mathscr{B}_{n}:$ The map $F_{n} \rightarrow G_{n}$ is given by $\bar{\beta}_{p^{n}} \rightarrow \beta_{p^{n}}$ and is an inclusion of Hopf algebras,

and consequently, $\lim _{n} G_{n}=R$ (on the identity $\beta_{0}$ ).

4. Proof of the Main Theorem. Setting $F=E_{*}\left(C P^{\infty}\right)$, it suffices to prove the following, purely algebraic

(4.1) THEOREM. With the notation and assumptions of $\$ 3$ :

(a) The elements $\beta_{1}, \beta_{p}, \cdots, \beta_{p^{n}}, \cdots$ form a free $R$-basis for $Q F$.

(b) $\beta_{p^{n}}^{p} \equiv s^{p^{n}} \beta_{p^{n}} \bmod \left(\beta_{1}, \cdots, \beta_{p^{n-1}}\right)$.

(c) If $\operatorname{deg}(x)<0$, then the elements $\beta_{1}, \beta_{p}, \cdots, \beta_{p^{n}}, \cdots$ form a simple system of generators for $F$.

Part (a) is immediate from diagram (3.5) and the definition of $F_{n}$. For part (b), pass to $G_{n}=F /\left(\beta_{1}, \cdots, \beta_{p^{n-1}}\right)$ and observe that $\beta_{p^{n}}^{p}=s^{p^{n}} \beta_{p^{n}}$ there. Part (c) requires induction upon $\operatorname{deg}\left(\beta_{j_{1}}^{t_{1}} \cdots \beta_{j_{k}}^{t_{k}}\right)$. Note that if $\operatorname{deg}(x)=0$, as in the case of complex $K$-theory, then the monomials $\beta_{j_{1}}^{t_{1}} \cdots \beta_{j_{k}}^{t_{k}}$ still provide a free $R$-basis for $F$, but the finiteness part of (2.1) is not satisfied.

5. Acknowledgments. Kamata [2] proves (2.2)(a) and part of (2.2)(c) for the case $E=M U Z_{p}$ and also has some results on $M U_{*}\left(C P^{\infty}\right)$. His techniques are quite different. The author is deeply grateful to Alex Zabrodsky for his generous advice and assistance.

\section{REFERENCES}

1. J. F. Adams, Quillen's work on formal groups and complex cobordism, Lectures, University of Chicago, Chicago, Ill., 1970.

2. M. Kamata, On the ring structure of $U_{*}(B U(1))$, Osaka J. Math. 7 (1970), 417-422. MR 43 \#1204.

3. D. Quillen, On the formal group laws of unoriented and complex cobordism theory, Bull. Amer. Math. Soc. 75 (1969), 1293-1298. MR 40 \#6565.

4. C. Schochet, On the structure of graded formal groups of finite characteristic, Proc. Cambridge Philos. Soc. (to appear).

5. G. W. Whitehead, Generalized homology theories, Trans. Amer. Math. Soc. 102 (1962), 227-283. MR 25 \#573.

Department of Mathematics, Hebrew University, Jerusalem, Israel

Current address: Department of Mathematics, Indiana University, Bloomington, Indiana 47401 